\title{
Garantie des soins médicaux de base: formes fructueuses et approches possibles
}

\section{Jürg Weber}

Spécialiste FMH en médecine générale, chargé de cours en médecine générale à l'Université de Berne

\section{* L'usage du masculin, respectivement du féminin répond ici exclusivement à des critères de lisibilité.}

Le rapport mentionné sur les modèles de soins peut être consulté sur le site web de la CDS sous la rubrique Structures ambulatoires.
Correspondance: Dr Jürg Weber Grundstrasse 3 $\mathrm{CH}-8556$ Wigoltingen juerg.weber[at]hin.ch
La Conférence suisse des directrices et directeurs cantonaux de la santé (CDS) et l'Office fédéral de la santé publique (OFSP) ont publié au printemps un rapport consacré aux «Nouveaux modèles de soins pour la médecine de premier recours». Le manque de médecins de famille se dessine d'une manière générale en Suisse et particulièrement dans les régions où la densité de population est faible. En vue de contribuer à prévenir ce manque à différents niveaux, une série d'articles présente diverses pistes de réflexion sous la forme d'approches possibles dont s'inspirer. Un premier modèle est présenté ci-après, qui est en cours de réalisation dans le canton de Thurgovie.

\section{Cabinet de groupe de médecins de campagne décentralisé et en réseau}

Dix cabinets avec quinze médecins de famille* participent actuellement à la mise en place d'un cabinet de groupe dans la région Thurtal - Untersee (cabinets des communes Felben-Wellhausen, Müllheim, Wigoltingen, Steckborn, Berlingen, Ermatingen). Ce modèle vise à garantir à moyen et long terme la médecine de famille et de garde dans les zones rurales et doit combattre le risque aigu d'un manque de médecins de premier recours dans la région, particulièrement en tenant compte des besoins et de l'environnement social de la prochaine génération de médecins de famille (travail à temps partiel, équilibre vie professionnelle / vie privée, etc.).

L'objectif est de maintenir dans les zones rurales des réseaux de cabinets grâce à des cabinets de groupe décentralisés. Le principe de la nouvelle approche est la mise à disposition gratuite de ressources et l'utilisation de synergies sous la forme d'un cabinet de groupe de médecins de famille décentralisé et en réseau, sur la base de processus qui existent et fonctionnent déjà. Dans le cabinet de groupe décentralisé, chacun des médecins participants gère bénévolement au moins un module du projet sous sa propre responsabilité et fournit les prestations qui y sont liées.

La base commune est la volonté de garantir 7 jours sur 7 et 24 heures par jour le service médical de garde par des visites à domicile dans tout le bassin de prise en charge. Le projet est coordonné par deux chefs de projet qui se partagent le travail organisationnel et administratif. De la sorte, seule une administration supplémentaire minimale est engendrée pour les cabinets. Chaque cabinet détermine à quels modules supplémentaires il participe activement ou passivement. Les cabinets demeurent économiquement et juridiquement autonomes.

Les modules souhaités et à exploiter prioritairement peuvent être choisis et réalisés en fonction des besoins régionaux. Voici trois exemples de modules: les soins d'urgence organisés de manière optimale avec prise en compte, dans la répartition du service, des besoins privés des médecins participants; la coordination régionale $\mathrm{du}$ «remplacement» des heures de consultation lors de longues absences; le module MPA-Wesen, où le médecin est déchargé de manière accrue par les assistantes médicales et une politique du personnel optimisée au plan régional est recher-

Série d'articles sur des modèles de cabinet de médecins de famille dans le «PrimaryCare»

Dans la revue de médecine de premier recours «PrimaryCare» paraît depuis février de cette année une série d'articles où sont présentés à intervalles irréguliers de nouveaux modèles de cabinet originaux, stimulants et efficaces.

A ce jour, les articles suivants sont parus et sont disponibles sur Internet à l'adresse www.primary-care.ch:

- Kissling B, Schilling G, Tschudi P. Le cabinet médical en tant qu'entreprise. PC 3/2012

- Koch W. Belle perspective d'avenir pour les médecins de premier recours. PC 3/2012

- Gantenbein M, Zeller A. Changement générationnel dans un cabinet médical - une situation gagnant-gagnant-gagnant. PC 11/2012

- Schaller P. Cité Générations. PC 12/2012

- Schindler A. «The spirit of Bubenberg» - la société Praxis Bubenberg SA à Berne est un cabinet de groupe géré en copropriété par ses collaborateurs. PC 12/2012

- Meer AE, Costea C, Wirthner A. Le cabinet en ligne - prise en charge des patients par Internet. PC 14/2012 
Objectifs et éléments-clés du cabinet de groupe décentralisé et en réseau

- Maintien des cabinets de médecine de famille dans les villages

- Recrutement de la succession par soi-même

- Décharge réciproque au quotidien (assistant au cabinet, assistante médicale*)

- Moyen terme entre autonomie et travail en équipe / mise en réseau

- Promotion du soutien par instances politiques et bailleurs de fonds

chée. Autre élément, l'attractivité de la profession de médecin de campagne doit être promue en particulier dans son propre cabinet (par ex. promotion ciblée de la relève sous la forme de stages d'introduction pour étudiants, formation d'assistants au cabinet, par la coopération avec les autorités locales et régionales, etc.).

Le cabinet de groupe décentralisé et en réseau doit se manifester vis-à-vis des autorités locales comme l'interlocuteur central pour toutes les questions de soins de santé dans les communes. A côté de l'information des autorités sur la profession et l'éventail de prestations des cabinets de médecins de campagne, une planification commune coordonnée doit en particulier se faire pour soutenir la prochaine génération de médecins de famille.

Aimeriez-vous présenter dans le cadre de notre série d'articles une autre initiative que vous connaissez visant à garantir et promouvoir la médecine de famille?

\section{Les modules suivants sont disponibles} au choix

- Soins d'urgence (service de garde commun dans les cabinets)

- Remplacement pendant les vacances

- Formation d'étudiants en médecine de famille

- Postes d'assistanat au cabinet

- Assistantes médicales (promotion, politique du personnel)

- «Spécialisation» des médecins et répartition réciproque

- Cercle de qualité/perfectionnement/revue de l'offre de perfectionnement

- Revue de presse

- Environnement professionnel (même niveau de connaissances/surplus de connaissances)

- Bulletin commun des cabinets

- Informatique, dossier électronique du patient, cybersanté,

- Pharmacie au cabinet / remise directe de médicaments

- Relations publiques / politique / aspects financiers

- Baisse des frais du cabinet / synergies / détecter les pertes

- Recherche au cabinet

- Contact avec les instituts de médecine de famille dans les universités

Correspondance: Sibylle Christen, Conférence des directeurs de la santé CDS, Maison des cantons, Speichergasse 6, Case postale 684, CH-3000 Berne 7, tél. 031 3562020 , sibylle.christen[at]gdk-cds.ch 